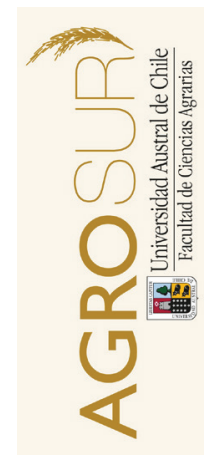

\title{
Variabilidad temporal de las propiedades físico-mecánicas de un suelo derivado de cenizas volcánicas bajo labranza convencional
}

\author{
Temporal variability of the physico-mechanical properties \\ of a volcanic ash soil under conventional tillage \\ Vásquez, N. ${ }^{a *}$, Salazar, F. ${ }^{b}$, Dörner, J. ${ }^{a, c}$ \\ ${ }^{a}$ Instituto de Ingeniería Agraria y Suelos, Facultad de Ciencias Agrarias, \\ Universidad Austral de Chile. Casilla 567, Valdivia, Chile. \\ ${ }^{b}$ Centro Regional de Investigación INIA Remehue. \\ ${ }^{c}$ Centro de Investigación en Suelos Volcánicos, Universidad Austral de Chile, Valdivia, Chile.
}

\begin{tabular}{l} 
A R T I C L E I N F O \\
\hline Keywords: \\
Andisol \\
Soil tillage \\
Mechanical properties \\
Wetting and drying cycles \\
\hline Original Research Article, \\
Soil Science \\
\hline *Corresponding author: \\
Nelson Vásquez \\
E-mail address: \\
josedorner@uach.cl, \\
nelson.vasquez.it@gmail.com
\end{tabular}

\begin{abstract}
A B S T R A C T
In order to determine the effect of soil tillage and natural processes of re-structuration that occur in a Andisol during a wheat growing season, this study evaluated the temporal variability of the physicomechanical properties of a Andisol under wheat crop. The assesment was carried out using both laboratory and in situ measurements to compare the registered values with those described in the literature as critical values for correct plant grow. The laboratory measurements (precompression stress, cohesion, air conductivity and bulk density) required undisturbed samples which were collected in $110 \mathrm{~cm}^{3}$ cylinders, at $5 \mathrm{~cm}$ depth, 10 times during the crop development. For the field measurements, the evolution of the moisture content and penetration resistance of the soil, as well as rainfall and soil temperature were recorded.

The soil tillage and the wetting and drying cycles that occurred during the study generated temporal changes in soil structure, which were expressed in the evaluated properties. Soil tillage, caused a decrease in the values of mechanical strength and bulk density, and increased the air conductivity and air capacity. After the traffic of the roller, the opposite effect was observed but without reaching the values of the initial state of the soil. Those values were reached during some events in the summer period, after several wetting and drying cycles. After grazing, no changes in the studied properties were observed. Only air conductivity exceeded the critical values described in the literature, however, due to the dynamic nature of soil structure, the soil was able to recuperate their functional attributes due to the high resilience of soils derived from volcanic ashes.
\end{abstract}

\section{RESUMEN}

Con el fin de conocer el efecto de la labranza y de los procesos naturales de estructuración que ocurren en un suelo derivado de cenizas volcánicas durante una temporada de cultivo, en este trabajo se estudió la variación temporal de las propiedades físico-mecánicas de un Andisol bajo cultivo de trigo. Esto fue determinado a través de mediciones en laboratorio e in situ, para comparar los valores determinados con aquellos descritos en la literatura como críticos para el correcto desarrollo de las plantas. Para las mediciones en laboratorio de capacidad de soporte, cohesión, conductividad de aire y densidad aparente, se recolectaron muestras no disturbadas, en cilindros de $110 \mathrm{~cm}^{3}$, a $5 \mathrm{~cm}$ de profundidad durante 10 periodos del cultivo. Con mediciones de campo, se registró la evolución del contenido de agua y la resistencia a la penetración del suelo, además de las precipitaciones y la temperatura del suelo.

La preparación de suelo y los ciclos de humectación y secado que ocurrieron durante el ensayo, generaron cambios temporales en la estructura del suelo, los que se manifestaron en las distintas propiedades evaluadas. La labranza, provocó una disminución de los valores de resistencia mecánica y densidad aparente, y aumentó los de conductividad de aire y capacidad de aire. Luego del paso del rodillo, se observó el efecto contrario pero sin alcanzar los valores del estado inicial del suelo. Estos se alcanzaron durante algunos de los muestreos hechos en el periodo estival, luego de varios ciclos de humectación y secado. Como consecuencia del pastoreo, no se evidenciaron cambios en las propiedades estudiadas. Solo la conductividad de aire superó los valores críticos descritos en la literatura; sin embargo, debido a la naturaleza dinámica de la estructura del suelo, éste recuperó sus atributos funcionales debido a la alta resiliencia de los suelos derivados de cenizas volcánicas.

Palabras clave: Andisol, labranza, propiedades mecánicas, ciclos de humectación y secado. 


\section{INTRODUCCIÓN}

La estructura del suelo se define como la organización o disposición de las partículas de suelo (Hillel, 1998). Este sistema está en un constante equilibrio dinámico, ya que la estructura se forma, se desintegra y se reforma de manera periódica dependiendo de los procesos hidráulicos y mecánicos a los que está sometido el suelo, influyendo sobre sus propiedades y sus funciones (Horn y Baumgartl, 1999).

La variación estacional que ocurre en la estructura del suelo, se debe principalmente a procesos internos (relacionado a los procesos hidráulicos como los ciclos de hinchamiento y contracción) y a las presiones externas que ocurren en el suelo (relacionado con el pisoteo animal o el tránsito de maquinaria), relacionados al manejo antrópico (Ellies et al., 1994a). A su vez, la persistencia de la estructura a través del tiempo depende de la estabilidad y la resistencia que presente el suelo ante agentes externos (Horn, 1988); así, cuando una presión supera a la resistencia del suelo, se alteran sus propiedades estructurales, químicas y biológicas (Ellies y Dörner, 1999).

Las propiedades de resistencia del suelo, cuantifican mecánicamente la funcionalidad del mismo, esto mediante los parámetros de intensidad, los cuales son utilizados para definir tanto las propiedades básicas del suelo como su funcionamiento. Los parámetros de intensidad son necesarios debido al dinamismo del suelo, ya que establecen los límites máximos a los que puede llegar el suelo sin afectar su funcionalidad. Así, el comportamiento mecánico de un suelo (cambio de volumen y resistencia al cizallamiento) puede ser descrito en términos del estado de tensión del suelo, en el cual la transmisión del estrés depende de los fluidos como el agua y el aire existentes y se puede manifestar en sus tres dimensiones, como rotación, compactación y descompactación y cizallamiento o corte (Horn y Baumgartl, 1999).

Existen muchos estudios que han analizado la variabilidad temporal de la estructura del suelo a través de sus propiedades hidráulicas y mecánicas (Horn, 2004; Osunbitan et al., 2005; Moret y Arrúe, 2007; Atkinson et al., 2009; Schwen et al., 2011). Sin embargo, estos estudios se han hecho en suelos que presentan una mineralogía cristalina, difiriendo de las características propias de los Andisoles, los cuales poseen una mineralogía no cristalina dominada por una arcilla de tipo alofán, lo que le confiere algunas particularidades como: una gran porosidad total y una densidad aparente $\leq 0,9 \mathrm{~g} \mathrm{~cm}^{-3}$ (Soil Survey Staff, 2006), además de una alta conductividad hidráulica (Ellies et al., 1997; Dörner et al., 2010a) y una gran capacidad de contracción y de resiliencia (Dörner et al., 2009a, 2009b, 2011).

En estos suelos, existen estudios que evalúan el efecto del manejo y el cambio de uso del suelo sobre el funcionamiento del sistema poroso del mismo (Ellies et al., 1997, 2000; Seguel et al., 2002; Bachmann et al., 2006; Dörner et al., 2009b, 2011). Sin embargo, son escasos los trabajos que analizan la variabilidad temporal de las propiedades que dependen de la estructura del suelo (Dörner et al., 2012; Dec et al., 2011, 2012; Ellies et al., 1993, 1994b). Por ejemplo, Ellies et al. (1993, 1994b), estudiaron la variación de las propiedades estructurales en dos estaciones distintas del año (invierno y verano) en suelos con distintos usos agropecuarios. Los autores concluyeron que el funcionamiento físico de los poros del suelo (resistencia mecánica y distribución de poros) cambia estacionalmente dependiendo más del tipo de suelo que del uso. Por otro lado, Dec et al. $(2011,2012)$, estudiaron la variabilidad temporal de la estructura del suelo bajo una condición de pastoreo, mostrando que existen cambios en las propiedades mecánicas e hidráulicas del suelo por efecto de los estreses producidos durante el pisoteo de los animales en pastoreo y los ciclos de humectación y secado. En este contexto, no existen trabajos que profundicen el análisis del efecto de la preparación de suelo y el establecimiento de un cultivo sobre la resistencia mecánica de los suelos volcánicos de la zona sur del país. Es por esto que el objetivo de este trabajo fue analizar la variabilidad temporal de las propiedades físico-mecánicas de un Andisol durante la temporada de un cultivo de trigo de invierno y, además, comparar los valores determinados con aquellos descritos en la literatura como críticos para el correcto desarrollo de las plantas.

\section{MATERIAL Y MÉTODOS}

\section{Clima, suelo y manejo}

El ensayo se realizó en el sur de Chile, en el Centro Regional de Investigación INIA Remehue, ubicado a 8 kilómetros al norte de Osorno (coordenada UTM 5.512.304,09 m Sur y 664.081,45 m Este, Datum WGS84). El sector de estudio pertenece al agroclima Osorno, el cual posee un clima mediterráneo frío, en donde la temperatura media anual es de $11,4^{\circ} \mathrm{C}$, presentando una máxima media en el mes de Enero de $23,8^{\circ} \mathrm{C}$ y una mínima media en el mes de Junio de $3,2^{\circ} \mathrm{C}$. El régimen de precipitaciones varía de $253,7 \mathrm{~mm}$ como máxima en el mes de Mayo a $50 \mathrm{~mm}$ como mínima en el mes de Febrero, presentando una media anual de $1.383 \mathrm{~mm}$ (Novoa y Villaseca, 1989).

El suelo corresponde a un Andisol perteneciente a la Serie Osorno (Typic Hapludand, CIREN, 2003), el cual se define como un suelo derivado de cenizas volcánicas recientes, que se ubica a una altura entre los 90 y 150 m.s.n.m. presenta una topografía ondulada, con pendiente dominante de 2 a $5 \%$, la profundidad del suelo varía de los 60 a $120 \mathrm{~cm}$ limitada por una capa de toba 
volcánica semi compactada. La textura se encuentra entre franco limosa a franco arcillo limosa (IREN, 1964).

El área del ensayo corresponde a un terreno de $2540 \mathrm{~m}^{2}$ que por 15 años se utilizó como pradera para pastoreo el cual fue labrado para el establecimiento de un cultivo de trigo de invierno (Triticum aestivum L. var. Kumpa-INIA). En Mayo de 2009, el suelo fue labrado 5 veces con una rastra off-set a $20 \mathrm{~cm}$ de profundidad, finalizando con una pasada de vibrocultivador. Tres días después de la labranza, se sembró el trigo en una dosis de $150 \mathrm{~kg} \mathrm{ha}^{-1}$, terminando las labores de preparación de suelo con el paso de un rodillo compactador (1500 kg de peso y ancho de operación de $300 \mathrm{~cm}$ ), siendo ésta la última alteración mecánica del suelo por el uso de maquinaria. A inicios de mayo de 2010, el trigo se pastoreó con vacas lecheras de aproximadamente 500 kg de peso por un periodo de 3 días y posterior a ello se realizó el último muestreo.

\section{Mediciones de campo y laboratorio}

Se instaló un datalogger con el que se almacenó la información proveniente de sensores ubicados a 5, 20 y $50 \mathrm{~cm}$ de profundidad, midiendo el contenido volumétrico de agua (TDR SM200) y la temperatura del suelo (termistores pt-100), junto con un pluviómetro para registrar las precipitaciones en el lugar. Las mediciones se realizaron desde inicios de junio de 2009, posterior a la siembra, hasta mayo de 2010 , fecha en que se realizó el último muestreo de suelo.

En distintos periodos del ensayo, tomando en cuenta tanto el efecto de la labranza como los ciclos natu- rales del suelo (Cuadro 1), se realizaron mediciones in situ a $10 \mathrm{~cm}$ de profundidad para determinar la resistencia a la penetración (RP) utilizando un penetrómetro estático (06.01 Hand Penetrometer Eijekelkamp Agrisearch Equipment, Giesbeek, The Netherlands) y el contenido volumétrico de agua $\left(\theta_{\text {vol. }}\right)$, mediante el uso de un TDR (TDR 200 Soil Multimeter FOM/mts, Easy Test, Lublin, Poland), el cual se calibró de acuerdo a lo propuesto en el manual de usuario 1.1 SM200 (Delta-T Devices Ltda, 2006). Además, se tomaron muestras no disturbadas de suelo de 2 a $7 \mathrm{~cm}$ de profundidad, en cilindros de $110 \mathrm{~cm}^{3}$ para los análisis de laboratorio, las cuales fueron utilizadas para los ensayos de resistencia al corte, capacidad de soporte, conductividad de aire y densidad aparente.

\section{Caracterización general del suelo}

Se determinó la textura del suelo (Forsythe, 1974), el contenido de alofán (Mizota y van Reeuwijk, 1989), la densidad real (Forsythe, 1974) y la materia orgánica (Sadzawka et al., 2004), cada uno con 3 repeticiones. Además, a partir de muestras no disturbadas de suelo colectadas en cilindros de $220 \mathrm{~cm}^{3}(\mathrm{n}=7)$ se registró la porosidad total y en cilindros de $110 \mathrm{~cm}^{3}(\mathrm{n}=18)$ se determinó la densidad aparente (Sandoval et al., 2012).

\section{Pretratamiento de las muestras}

Antes del ensayo de resistencia al corte $(n=10)$ y capacidad de soporte $(n=4)$, las muestras fueron saturadas en una bandeja con agua mediante capilaridad

Cuadro 1. Época de toma de muestras, condición de suelo y evolución del contenido de agua $(0-10 \mathrm{~cm})$ base volumen $\left(\theta_{\text {vol. }}\right)$ medido con TDR durante el ensayo.

Table 1. Soil sampling events, soil condition and evolution of the volumetric water content $(0-10 \mathrm{~cm})$ measured with TDR during the test $\left(\theta_{\text {vol. }}\right)$.

\begin{tabular}{|c|c|c|c|c|c|}
\hline Fecha & Muestreo & Condición de suelo & \multicolumn{3}{|c|}{$\begin{array}{l}\theta_{\text {vol. }} \\
(\%)\end{array}$} \\
\hline 14 abr. 2009 & PD & Pradera degradada & 21,9 & \pm & 0,2 \\
\hline 29 may. 2009 & SP & Suelo preparado & 53,9 & \pm & 0,5 \\
\hline 01 jun. 2009 & SS1 & Suelo sembrado & 57,9 & \pm & 0,3 \\
\hline 08 sept. 2009 & SS2 & Suelo sembrado * & 60,6 & \pm & 0,3 \\
\hline 20 oct. 2009 & SS3 & Suelo sembrado* & 49,0 & \pm & 0,3 \\
\hline 06 dic. 2009 & SS4 & Suelo sembrado* & 43,6 & \pm & 0,4 \\
\hline 24 ene. 2009 & SS5 & Suelo sembrado * & 37,1 & \pm & 0,3 \\
\hline 07 mar. 2010 & SS6 & Suelo sembrado* & 43,4 & \pm & 0,4 \\
\hline 22 abr. 2010 & SS7 & Suelo sembrado* & 54,5 & \pm & 0,5 \\
\hline 06 may. 2010 & SS8 & Después de la entrada de animales & 49,7 & \pm & 0,5 \\
\hline
\end{tabular}

* En estas etapas no hubo intervención mecánica sobre el suelo. $\theta_{\text {vol. }}$ (n=176). Valores promedio \pm 1 error estándar. 
por al menos 72 horas, para así asegurar una tensión de agua $<1 \mathrm{hPa}$ (Hartge y Horn, 2009). Luego, se equilibraron a una tensión de $60 \mathrm{hPa}$ (capacidad de aire del suelo) en una bandeja de arena por 7 días, registrando la masa en una balanza de precisión (Precisa 0,01 g). Además, cuatro muestras con la humedad de terreno fueron utilizadas para definir su capacidad de soporte.

\section{Propiedades del suelo}

Una vez equilibradas las muestras, se midió la conductividad de aire $(n=14)$ y se realizaron los ensayos de consolidación confinada con drenaje libre (Hartge, 2000) y la prueba de corte directo (Kézdi, 1980).

A partir del peso de las muestras se determinó la capacidad de aire (CA) $(n=14)$, la que se calculó mediante la diferencia entre la porosidad total y el contenido volumétrico de agua determinado a $60 \mathrm{hPa}$ de tensión de agua (Dec et al., 2012). La conductividad de aire (k) se determinó usando un flujómetro de aire, en donde se aplica una diferencia de presión menor a $1 \mathrm{hPa}$ para permitir un flujo laminar ascendente de aire a través de la muestra de suelo. Cada medición se repitió 3 veces a la misma diferencia de presión. Durante las mediciones se registraron los cambios en la presión de aire y la temperatura, los que son considerados en los siguientes cálculos (Dörner y Horn, 2006):

$$
\mathrm{k}_{1}=\rho_{1} * \mathrm{~g} *\left(\frac{\Delta \mathrm{V} * \mathrm{~L}}{\Delta \mathrm{t} * \Delta \mathrm{p} * \mathrm{~A}}\right)
$$

Donde, $\mathrm{k}_{1}$ : conductividad de aire $\left(\mathrm{m} \mathrm{s}^{-1}\right) ; \rho_{\mathrm{l}}$ : densidad del aire durante la medición $\left(\mathrm{kg} \mathrm{m}^{-3}\right)$; g: aceleración de gravedad $\left(9,81 \mathrm{~m} \mathrm{~s}^{-2}\right) ; \Delta V$ : volumen de aire que fluye en la muestra durante el tiempo $\Delta \mathrm{t}(\mathrm{min})$; L: longitud del cilindro (m); $\Delta \mathrm{p}$ : presión (hPa); A: área del cilindro $\left(\mathrm{m}^{2}\right)$.

El ensayo de consolidación se realizó en un odómetro (T-Controls), utilizando cargas normales acumulativas de 6,$25 ; 12,5 ; 25 ; 50 ; 100 ; 200$ y $400 \mathrm{kPa}$, cada 6 minutos. Con los datos obtenidos se calculó el valor de preconsolidación (Pv) o capacidad de soporte mediante el método de Casagrande, utilizando la metodología matemática propuesta por Baumgartl y Köck (2004), mediante el uso del software RETC versión 6.02 (van Genuchten et al., 1991).

El ensayo de resistencia al corte se realizó en una máquina de corte directo (T-Controls), en las que se aplicaron cargas normales de 13,2; 25; 50; 75; 100; 150; 300 y $400 \mathrm{kPa}$ con repetición en 25 y $150 \mathrm{kPa}$. Transcurridos $20 \mathrm{~min}$, necesarios para equilibrar las presiones en el suelo, se realizaron mediciones cada $5 \mathrm{~min}$. Mediante el ensayo de corte se puede conocer la cohesión del suelo, la cual se determinó mediante la recta de Coulomb (Kézdi, 1980):

$$
\tau=\tan \varphi^{*} \sigma_{\mathrm{n}}+\mathrm{c}
$$

Donde, $\tau$ : resistencia al corte $(\mathrm{kPa})$; $\varphi$ : ángulo de roce interno $\left({ }^{\circ}\right) ; \sigma_{\mathrm{n}}$ : carga normal $(\mathrm{kPa})$; c: cohesión $(\mathrm{kPa})$

Una vez terminado el ensayo de consolidación y corte, se determinó su densidad aparente $(n=18)$ (Sandoval et al., 2012).

\section{Análisis estadístico}

Como la capacidad de soporte y la conductividad de aire no presentaron una distribución normal, se transformó a logaritmo tal como lo sugieren Hartge y Horn (2009). Para el análisis entre muestreos se realizó un análisis de varianza y en el caso de encontrar diferencias se aplicó un test de Tukey con un 95\% de confianza. Para los datos que no pudieron ser normalizados (RP y $\theta_{\text {vol }}$.), se aplicó el test de Kruskal-Wallis y en el caso de encontrar diferencias se aplicó el test de Dunn con un 95\% de confianza.

\section{RESULTADOS Y DISCUSIÓN}

\section{Características generales del suelo}

El Cuadro 2 presenta la caracterización general del suelo en estudio a tres distintas profundidades. Según IREN (1964), la Serie Osorno presenta una textura de tipo franco limosa. La densidad real y la estructura corresponden a lo descrito por IREN-UACH (1978). Los contenidos de alofán son similares a los obtenidos para otros Andisoles (Typic Hapludand), sin embargo, no se observó un aumento de este mineral en profundidad como lo determinado en otros estudios (Huygens et al., 2005; Dörner et al., 2009b).

Propiedades sensibles al manejo del suelo como la densidad aparente, la porosidad total y la materia orgánica (Cuadro 2) presentaron valores distintos a los reportados por IREN-UACH (1978). Sin embargo, en un sistema de pradera degradada es esperable que en el tiempo se produzca un aumento en la densidad aparente, con la consiguiente disminución de la porosidad total, especialmente de la macroporosidad, y una evidente reducción en el contenido total de la materia orgánica (Ellies et al., 1996; Dörner et al., 2009b). Aún así, el suelo del estudio presentó una buena estructura, granular en superficie y de bloques sub angulares en profundidad.

\section{Evolución de las condiciones de humedad y temperatura del suelo durante la temporada de cultivo}

Los datos obtenidos por la estación de monitoreo correspondientes a precipitaciones, temperatura del suelo y contenido volumétrico de agua asociado al sitio experimental se presentan en la Figura 1. 
Cuadro 2. Caracterización general del suelo a distintas profundidades. (Arena: $\phi>2000$ - 63 mm, Limo: $\phi 63$ - 2 mm, Arcilla: $\phi<2$ mm, M.O.: Materia orgánica, PT: Porosidad total, Dr: Densidad real, Da: Densidad aparente).

Table 2. General characteristics of the soil at different depths. (Sand: $\phi>2000-63 \mathrm{~mm}$, Loam: $\phi 63-2 \mathrm{~mm}$, Clay: $\phi<2 \mathrm{~mm}$, M.O.: Organic matter, PT: Total porosity, Dr: Particle density, Da: Bulk density).

\begin{tabular}{|c|c|c|c|c|c|c|c|c|c|c|}
\hline $\begin{array}{l}\text { Profundidad } \\
\text { (cm) }\end{array}$ & Textura & Arena & $\begin{array}{l}\text { Limo } \\
0(\%\end{array}$ & $\begin{array}{l}\text { Arcilla } \\
\text { 0) }\end{array}$ & Alofán & Estructura & $\begin{array}{l}\text { M.0. } \\
---\quad \text { (\% }\end{array}$ & $\begin{array}{l}\text { PT } \\
----\end{array}$ & $\begin{array}{l}\text { Dr } \\
---(g \text { cn }\end{array}$ & $\begin{array}{c}\text { Da } \\
\left.\text { m }^{-3}\right)---\end{array}$ \\
\hline 5 & $\begin{array}{l}\text { Franco } \\
\text { Limosa }\end{array}$ & $14,5 \pm 2,8$ & $60,2 \pm 2,3$ & $25,3 \pm 1,8$ & $8,2 \pm 0,5$ & Granular & $12,6 \pm 0,1$ & $66,4 \pm 0,1$ & $2,44 \pm 0,02$ & $0,83 \pm 0,02$ \\
\hline 20 & $\begin{array}{l}\text { Franco } \\
\text { Limosa }\end{array}$ & $20,5 \pm 2,0$ & $55,1 \pm 3,2$ & $24,5 \pm 1,4$ & $5,7 \pm 0,6$ & Subangular & $11,8 \pm 0,1$ & $56,2 \pm 0,7$ & $2,25 \pm 0,01$ & $0,96 \pm 0,02$ \\
\hline 50 & Franca & $33,1 \pm 1,4$ & $49,8 \pm 2,3$ & $17,0 \pm 0,9$ & $8,3 \pm 1,0$ & Subangular & $4,8 \pm 0,1$ & $58,3 \pm 0,6$ & $2,2 \pm 0,03$ & $0,91 \pm 0,01$ \\
\hline
\end{tabular}

Valores promedio \pm 1 error estándar.

La distribución de precipitaciones y temperatura del suelo muestran una marcada estacionalidad (Figura 1a, b), siendo, el monto de las precipitaciones, mayores durante los meses de invierno y menores en la época estival; lo contrario se observó para la temperatura del suelo, la cual está relacionada con el régimen de temperaturas ambientales (Novoa y Villaseca, 1989). Esto es válido para las tres profundidades de muestreo, presentando la temperatura una menor fluctuación a medida que aumenta la profundidad.

Los registros del TDR medidos a $10 \mathrm{~cm}$ de profundidad (Cuadro 1), concuerdan con los resultados del contenido de agua base volumen $\left(\theta_{\text {vol. }}\right)$ determinados por la estación de monitoreo (Figura 1c), los cuales a su vez se relacionan directamente con el régimen de precipitaciones. De acuerdo a Dörner et al. (2012), el contenido volumétrico de agua del suelo a 60 y 15430 hPa de tensión fluctúan entre 62 - 50\% y 32 - 25\%, respectivamente. Los máximos contenidos de agua en el suelo están en los muestreos de otoño (SS1), invierno (SS2) y principios de primavera (SS3) (Cuadro 1), lo cual al comparar con las precipitaciones, se puede ver claramente que éstas son elevadas en los meses de junio, julio y septiembre, mientras que los menores contenidos de agua se producen en los meses de abril del 2009 (PD) y enero del 2010 (SS5), en donde las precipitaciones son bajas y el agua se pierde rápidamente por evapotranspiración, ya que las temperaturas, tanto del suelo como del aire, son más elevadas.

Efecto de la preparación de suelo y de los ciclos de humectación y secado sobre las propiedades de resistencia mecánica del suelo: Resistencia a la penetración (RP), cohesión y capacidad de soporte (Pv)

La preparación de suelos provocó que los valores de resistencia mecánica determinados tanto con humedad de terreno como a $60 \mathrm{hPa}$ de tensión de agua, disminuyan considerablemente en el paso de PD a SP (p. ej. de $2727 \mathrm{kPa}$ a $1552 \mathrm{kPa}$ para RP), siendo este el efecto más claro que tuvo el suelo durante el ensayo (Figura 2).

La resistencia a la penetración superó en tres ocasiones los $2000 \mathrm{kPa}$ (PD, SS5 y SS6) y el valor de preconsolidación a humedad de terreno $\left(\mathrm{Pv}_{\text {(Terreno) }}\right)$ superó en una ocasión los $90 \mathrm{kPa}$ (PD), siendo estos valores señalados como críticos según lo propuesto por Horn y Fleige (2009), y que evidencian problemas de compactación de suelos cuando es determinado a un contenido volumétrico de agua del suelo equivalente a capacidad de campo. Sin embargo, en este estudio los valores altos se debieron a que el contenido volumétrico de agua del suelo $\left(\theta_{\mathrm{vol}}\right)$ en el momento de los muestreos (Cuadro 1), fueron menores que la humedad a capacidad de campo, que correspondió a 44\% en PD, $45 \%$ en SS5 y $47 \%$ en SS6 según lo reportado por Feest (2011) en un estudio paralelo a este. Por lo tanto, estos altos valores de RP no señalan problemas de compactación. Por otro lado, cuando el suelo alcanzó valores de $\theta_{\text {vol. }}$ cercanos a capacidad de campo, p. ej. en SS4 en la RP, el valor fue menor a $2000 \mathrm{kPa}$.

La labranza convencional tiende a generar agregados de menor tamaño respecto a la agregación natural, con un $50 \%$ de agregados menores a $5 \mathrm{~mm}$ de diámetro (Keller et al., 2007), tendiendo a la homogeneización del suelo (Martínez et al., 2008). Este efecto se debe al desmenuzamiento y pulverización del suelo, proceso durante el cual se rompen los agregados, disminuyendo su tamaño. Esto también provoca una disminución en la cantidad de puntos de contacto entre estos, disminuyendo así la resistencia del suelo (McVay et al., 2006; Seguel, 2006). Además, se observa un menor valor de densidad aparente (Figura 3a), lo que está relacionado a menores valores de resistencia mecánica (Ellies et al., 1996). Con respecto a esto, Navarro et al. (2000) afirman que cualquier sistema de labranza modifica la 
a)

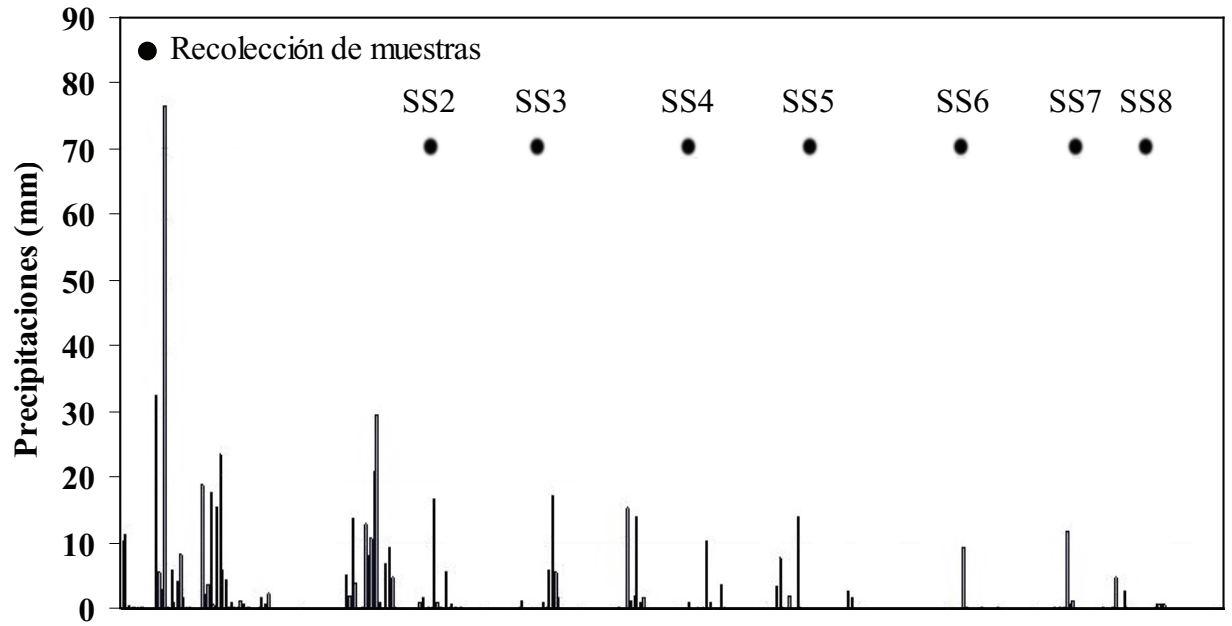

b)

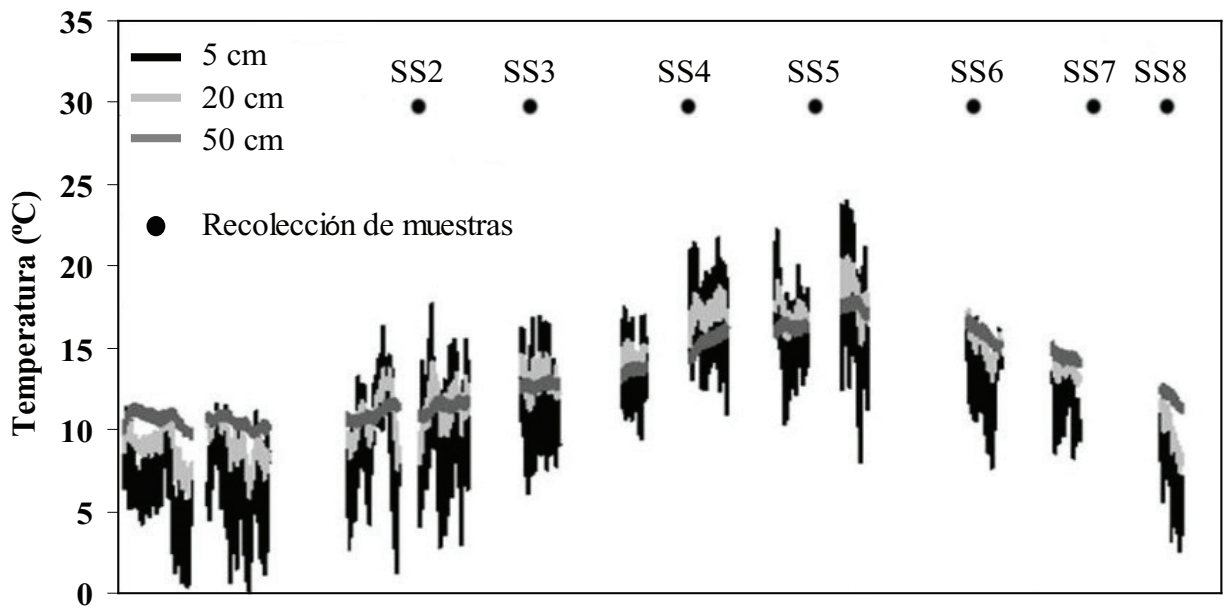

c)

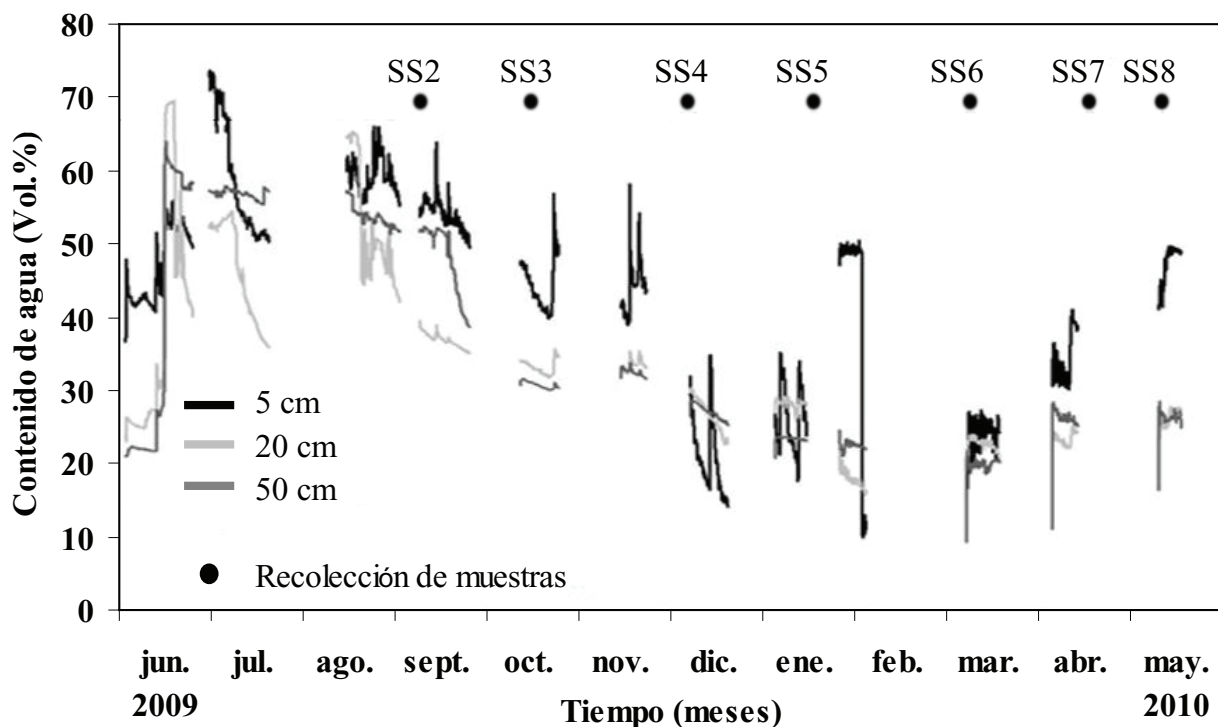

Figura 1. Evolución de a) Precipitaciones, b) Temperatura del suelo y c) Contenido de agua del suelo ( $\theta_{\text {vol. }}$ ) durante el estudio. Figure 1. Evolution of a) Rainfall, b) Soil temperature and c) soil water content $\left(\theta_{\text {vol. }}\right)$ during the test period. 


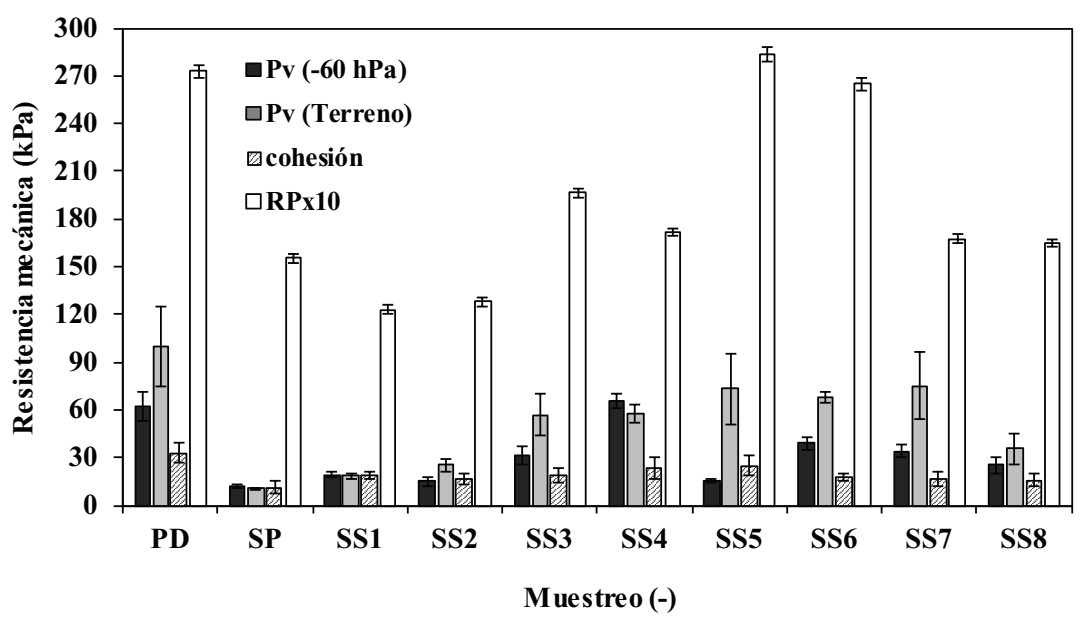

Figura 2. Valores de resistencia mecánica en función de la época de muestreo. Barras indican \pm 1 error estándar. Pv ${ }_{(-60 \mathrm{hPa})}$ : Capacidad de soporte a $60 \mathrm{hPa}$ de tensión de agua $(\mathrm{n}=4), \mathrm{Pv}_{(\text {Terreno) }}$ : Capacidad de soporte medido a humedad de terreno (n=4), cohesión ( $\mathrm{n}=10)$, RP: Resistencia a la penetración ( $\mathrm{n}=176)$.

Figure 2. Soil strength values as function of sampling dates. Bars indicate \pm 1 standard error. $\mathrm{Pv}_{(-60 \mathrm{hPa})}$ : precompression stress at $60 \mathrm{hPa}$ water tension $(\mathrm{n}=4), \mathrm{Pv}_{\text {(Terreno) }}$ : precompression stress measured at field soil moisture $(\mathrm{n}=4)$, cohesion $(\mathrm{n}=10), \mathrm{RP}$ : Penetration resistance $(n=176)$.

estructura del suelo, repercutiendo esto en sus propiedades mecánicas y densidad aparente, disminuyendo ambas al ser intervenido por labranza.

En cuanto a la RP y el $\mathrm{Pv}_{\text {(Terreno) }}$ (Figura 2), desde SS1 a SS8 existe una tendencia de que a medida que el contenido de agua del suelo disminuye (Cuadro 1) y la temperatura se eleva (Figura 1b), los valores de RP y $\mathrm{Pv}_{\text {(Terreno) }}$ tienden a aumentar, y luego con el aumento del contenido de agua en el suelo estas propiedades disminuyen su valor. Sin embargo, para $\mathrm{Pv}_{\text {(Terreno) }}$ estos cambios no fueron significativos ( $p>0,05$ ), lo que se debe a los altos valores de error estándar, especialmente en SS3, SS5 y SS7.

Así, luego de la reducción de la resistencia mecánica a la penetración debido a la labranza, se observa una segunda disminución significativa $(\mathrm{p} \leq 0,05)$ desde $\mathrm{SP}$ a SS1 (Figura 2), aun siendo posterior a la acción mecánica del rodillo compactador, lo que se asocia a la mayor humedad presente en el suelo en SS1 $\left(\theta_{\text {vol. }}=57,9 \%\right.$, Cuadro 1). Esto se debe a que el roce entre las partículas en un material más húmedo es menor por la lubricación que proporciona el agua (Ellies et al., 1993; Dec et al., 2011). Por otro lado $\mathrm{Pv}_{\text {(Terreno) }}$ aumentó su valor luego del paso del rodillo compactador, sin embargo, este aumento no fue significativo ( $p>0,05)$.

En el muestreo SS3, RP aumenta con respecto a SS2 ( $p \leq 0,05)$, debido a que se ubica al inicio de la primavera, provocando que exista una mayor intensidad de secado del suelo debido al aumento de la temperatura (Cuadro 1). Por otro lado, en esta estación se produce un mayor crecimiento por parte de la planta lo que trae consigo un incremento en la evapotranspiración y, por ende, una disminución en $\theta_{\text {vol. }}$ (Cuadro 1) y un aumento del $\mathrm{Pv}_{\text {(terreno) }}$ (Figura 2).

Dec et al. (2011), indican que un cambio en $\theta_{\text {vol. }}$ está estrechamente relacionado a un cambio en RP y que su aumento involucra también un aumento en Pv. Esta alza se observa nuevamente en los muestreos SS4 y SS5 $(\mathrm{p} \leq 0,05)$ por las condiciones climáticas propias del periodo estival y el efecto del mayor secado del suelo producto de la demanda de agua por parte del cultivo, las que provocan una mayor contracción del suelo (Feest, 2011), induciendo un aumento de los puntos de contacto entre los agregados, con el consecuente aumento del $\mathrm{Pv}_{\text {(terreno) }}$ y la RP (Figura 2). Por otro lado, SS4 disminuye con respecto a SS3 ( $p \leq 0,05)$, lo que se puede deber a una condición friable del suelo. Con respecto a esto Czarnes et al. (2000), señalan que la resistencia de un suelo homogeneizado (sin estructura) puede disminuir luego de ser aplicados varios ciclos de humectación y secado. Seguel (2006), explica que esta situación se debe a que al hacer la medición sobre la matriz del suelo y no sobre el agregado se toma en cuenta la porosidad formada en los procesos formadores de estructura, los que se convierten en planos de falla provocando un aumento en la friabilidad del suelo y disminuyendo su resistencia mecánica. Esta situación se podría asociar en cierta medida a lo que ocurre en el paso de SS3 a SS4 ya que, si bien, la preparación de suelo rompe la estructura no la elimina por completo.

En el paso de SS7 a SS8 no se evidenciaron cambios significativos en la RP y en el $\mathrm{Pv}_{\text {(terreno) }}(\mathrm{p}>0,05)$ lo que responde al clima propio de otoño, el cual produce un mayor humedecimiento del suelo (Cuadro 1), por lo 
tanto una mayor hinchazón provocando una disminución de éstas propiedades. A pesar de esto, no se evidenciaron cambios debido al efecto del pisoteo animal (Figura 2), ya que aún cuando el valor de preconsolidación fue de los más bajos dentro del estudio, lo que se asocia a una menor resistencia del suelo, las características intrínsecas de los Andisoles le confieren una alta elasticidad asociado a la materia orgánica y el contenido de alofán (Dörner et al., 2011).

En cuanto a las propiedades mecánicas determinadas con una tensión de agua de $-60 \mathrm{kPa}$, la cohesión interna del suelo y el $\mathrm{Pv}_{(--60 \mathrm{hPa})}$ obtuvieron sus valores más altos en PD (manejo de pradera degradada) con 33 kPa y $62 \mathrm{kPa}$, respectivamente. Por otro lado, los menores valores se obtuvieron en SP, con $11 \mathrm{kPa}$ para la cohesión y $12 \mathrm{kPa}$ para el $\mathrm{Pv}_{(-60 \mathrm{hPa})}$, debido al efecto de la preparación de suelo (Figura 2).

En SS1 aumentó como consecuencia del paso del rodillo compactador $(\mathrm{p} \leq 0,05)$, lo que produjo un asentamiento, perdiendo espacio poroso y aumentando los puntos de contacto. Esto provoca un mayor acercamiento y encaje entre las rugosidades de los agregados, explicando el aumento en la cohesión (Ellies et al., 1996; Dörner et al., 2009a). Por su parte, $\mathrm{Pv}_{(-60 \mathrm{hPa})}$ aumentó su valor luego del paso de rodillo pero este aumento no fue significativo ( $p>0,05)$.

Durante los ciclos de humectación y secado se aprecia una tendencia donde la cohesión interna del suelo registra desde SS2, un aumento hasta SS5, alcanzando un valor de $25 \mathrm{kPa}$ (Figura 2). Además, el alto valor encontrado en SS5 también pudo haber sido afectado por un mayor desarrollo de las raíces del trigo, especialmente en el periodo de floración del cultivo (Martínez et al., 2008) (cercano a SS5), lo que pudo haber producido una mayor agregación y estabilización de la estructura debido al mayor secado del suelo y entrelazamiento de las raíces (Horn y Smucker, 2005; Six et al., 2004). Con respecto a esto, Ellies et al. (2000), indican que las raíces finas pueden aumentar la resistencia del suelo por el efecto de secado que producen, traduciéndose esto en una mayor cohesión y preconsolidación del suelo. Sin embrago, al contrario de lo anterior, en el $\mathrm{Pv}_{(-60 \mathrm{hPa})}$ en el paso de SS4 a SS5, se observó una disminución significativa $(p \leq 0,05)$. Con respecto a esto Ellies et al. (2000), afirman que existe una relación inversa entre la macroporosidad y $\mathrm{Pv}$, ya que a mayor el volumen de macroporos menor es la preconsolidación del suelo. Feest (2011), muestra un aumento de la macroporosidad la cual aumentó $19 \%$ a $25 \%$ de SS4 a SS5 lo que puede explicar la disminución de $\mathrm{Pv}_{(-60 \mathrm{hPa})}$.

Tanto para la cohesión del suelo, desde SS5, como para el $\mathrm{Pv}_{(-60 \mathrm{hPa})}$ en SS6, los valores de éstas propiedades, disminuyeron hasta SS8 manteniendo lo esperado según las condiciones climáticas (Figura 1b,c). En ninguna de las propiedades medidas a $60 \mathrm{hPa}$ de tensión de agua se observaron variaciones con respecto al pisoteo animal.
En definitiva, la preparación de suelos bajo labranza tradicional en el corto plazo promueve una mayor porosidad (Figura 3) y una menor resistencia del suelo (Figura 2); sin embargo, la permanencia de este estado se ve afectada por los procesos naturales del suelo (ciclos de humectación y secado) y por el tiempo y tipo de uso, el cual tiende a estructurar nuevamente el suelo llevándolo hacia su situación original.

\section{Efecto de la preparación de suelo y ciclos de humectación y secado sobre el sistema poroso: densidad aparente (Da), capacidad de aire (CA) y conductividad de aire $\left(\mathrm{k}_{\mathbf{l}}\right)$}

Tanto la labranza como los ciclos de humectación y secado causaron cambios en su estabilidad mecánica, lo que influye a su vez sobre el sistema poroso (Ellies et al., 2000). En ese contexto, resulta relevante analizar cómo los cambios descritos influyen sobre parámetros de capacidad (densidad aparente y capacidad de aire) e intensidad (conductividad de aire), los que permiten comprender el funcionamiento del sistema poroso del suelo y su dinámica temporal (Figura 3).

La densidad aparente disminuyó de $0,78 \mathrm{~g} \mathrm{~cm}^{-3}$ en PD a $0,56 \mathrm{~g} \mathrm{~cm}^{-3}$ en SP $(\mathrm{p} \leq 0,05)$, debido a que la preparación de suelos provocó un aumento en el volumen del suelo por efecto del movimiento mecánico (Szűcs et al., 2012), lo que al mismo tiempo está relacionado a una mayor porosidad total (Ellies et al., 1996). Luego del paso del rodillo compactador, se observó un aumento de la Da en SS1 ( $\mathrm{p} \leq 0,05)$ ya que, tal como lo indican Ahmad et al. (2007), la porosidad total del suelo disminuye y la Da aumenta debido a la presión generada por el rodillo.

Durante los ciclos de humectación y secado, se observó un aumento de la densidad aparente desde SS2 a SS3 (Figura 3a) debido a la contracción del suelo producto de la disminución del $\theta_{\text {vol. }}$ de $60,6 \%$ en SS2 a 49,0\% en SS3 (Cuadro 1). Según Dörner et al. (2010a), la capacidad de contracción del suelo, que se puede medir a través del coeficiente de extensibilidad lineal (COLE), es mayor cuando disminuye la densidad aparente producto de una homogenización del suelo (p. ej. con la labranza). En ese contexto, la mayor magnitud de contracción se produce en los primeros ciclos de formación de estructura (Leiva, 2009), siendo estos representados en los muestreos SS2 y SS3 (Feest, 2011), los que explican el incremento en la Da. En los restantes ciclos de humectación y secado se observó una disminución de la Da con respecto a SS3, sin presentar una mayor variación desde SS4 hasta SS8, lo que se puede asociar a una mejor estabilización del suelo lo que provocaría una disminución en la Da (Semmel, 1990), siendo este efecto más pronunciado en SS5 asociado a una mayor porosidad total (Feest, 2011).

La capacidad de aire (CA) corresponde a la porosidad gruesa del suelo (poros de drenaje rápido), la cual 


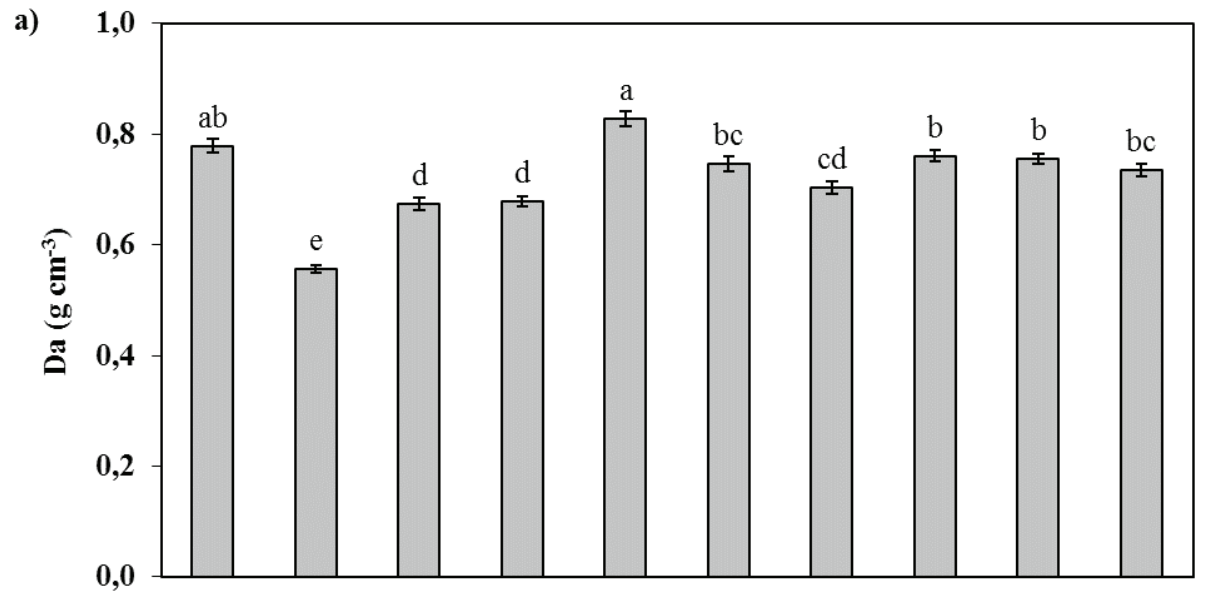

b)
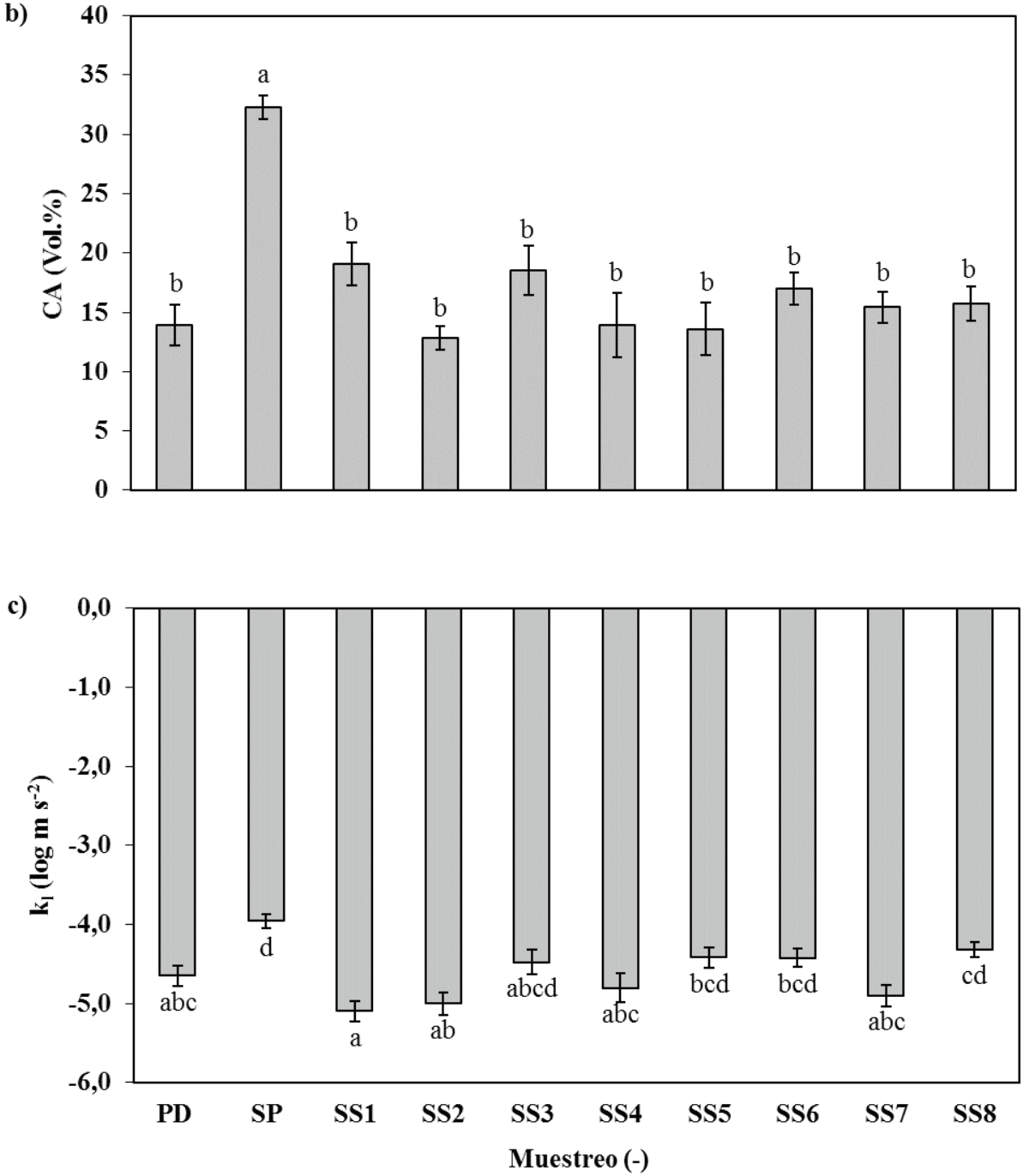

Figura 3. Variación de las propiedades relacionadas a la porosidad en función de la época de muestreo. Barras indican \pm 1 error estándar. a) Da: Densidad aparente (n=18), b) CA: Capacidad de aire $(\mathrm{n}=14), \mathrm{c}) \mathrm{k}_{\mathrm{l}}$ : Conductividad de aire $(\mathrm{n}=14)$.

Figure 3. Changes in properties related to soil porosity as function of sampling dates. Bars indicate \pm 1 standard error. a) Da: Bulk density ( $n=18)$, b) CA: Air capacity $(n=14)$, c) $k_{1}$ : Air conductivity $(n=14)$. 
depende de la porosidad inter-agregado que es fuertemente susceptible a la deformación (Dec et al., 2011). La preparación de suelo provocó un aumento significativo de esta propiedad ( $\mathrm{p} \leq 0,05$ ) de 13,9\% en PD a $32,3 \%$ en SP (Figura 3b). Posteriormente, disminuyó en SS1 a 19,1\% SP (p $\leq 0,05)$ luego del paso de rodillo que produjo la compactación del suelo. En los ciclos de humectación y secado (desde SS2 a SS8), no se evidenciaron cambios significativos en CA ( $p>0,05)$, lo que se debe a que los procesos de formación de estructura provocan una alta estabilidad estructural, lo que repercute en una baja variación de la porosidad gruesa (Dörner et al., 2009c). En este contexto, el valor más bajo de CA se produjo en SS2, alcanzando un valor de 12,8\% el cual se encuentra por encima del considerado como crítico para esta propiedad ( $<8 \%$, según lo propuesto por Horn y Fleige, 2009), por lo tanto, no deberían haber problemas de renovación de aire del suelo.

La preparación de suelos provocó un aumento significativo $(\mathrm{p} \leq 0,05)$ de la conductividad de aire $\left(\mathrm{k}_{\mathrm{l}}\right)$, de $-4,65 \log \mathrm{m} \mathrm{s}^{-2}$ en PD a $-3,96 \log \mathrm{m} \mathrm{s}^{-2}$ en SP (Figura 3c), debido al aumento de la macroporosidad (Feest, 2011) y la capacidad de aire (Figura 3 b). En ese contexto, $\mathrm{k}_{1}$ además de depender del volumen de macroporos del suelo, está influenciada por la organización espacial de los agregados, lo que determina aspectos geométricos del medio poroso como la continuidad, tortuosidad e interconexión de los poros (Moldrup et al., 2003; Dörner y Horn, 2006). Por lo tanto, el incremento de $\mathrm{k}_{\mathrm{l}}$ luego de la preparación de suelo, también está asociada a un incremento en la continuidad e interconexión de los poros debido a la menor cantidad de puntos de contacto entre los agregados (Seguel, 2006). La compactación por el paso del rodillo provocó una pérdida en la macroporosidad y, consecuentemente, una menor aireación (Ball et al., 1997), disminuyendo $\mathrm{k}_{1}$ a -5,10 log $\mathrm{m} \mathrm{s}^{-2}$ en SS1 y -5,00 log $\mathrm{m} \mathrm{s}^{-2}$ en SS2 (Figura 3c). En ese contexto, $\mathrm{k}_{1}$ supera el valor crítico correspondiente a < $-4,92 \log \mathrm{m} \mathrm{s}^{-1}$ (Horn y Fleige, 2009) lo que indica problemas de compactación de suelo que pueden redundar en un deficiente intercambio gaseoso.

El comportamiento de la conductividad de aire en el tiempo es consistente con lo señalado por Alaoui et al. (2011), quienes indican que la compactación, el cizallamiento y la reorganización de los agregados del suelo debido a la contracción, puede afectar en gran medida la geometría de los poros de los suelos agrícolas y en consecuencia $\mathrm{k}_{\mathrm{l}}$. En ese contexto, los ciclos de humectación y secado contribuyeron a un aumento de $\mathrm{k}_{1}$ luego del paso de rodillo compactador (Figura 3c), siendo más estable en el tiempo, debido a la formación grietas durante el secado del suelo desde SS2 a SS6 (Figura 1), lo que produce una mayor continuidad entre los poros, favoreciendo el flujo de aire en el suelo (Dörner et al., 2011). También es necesario considerar el efecto del carbono orgánico, que actúa como un elemento elástico que permite la recuperación volumétrica y funcional de los poros del suelo (Dörner et al., 2009b), la actividad biológica y la creación de bioporos (Ellies et al., 1997) y las raíces de las plantas (Horn y Smucker, 2005), que promueven una mayor conectividad entre los poros del suelo, lo que provoca un mayor estabilidad en $\mathrm{k}_{\mathrm{l}}$, no registrándose variaciones significativas $(p>0,05)$ desde SS3 en adelante.

En relación al pisoteo animal, como consecuencia del pastoreo del trigo, no se encontraron diferencias significativas $(\mathrm{p}>0,05)$ en ninguno de los parámetros asociados al sistema poroso (Figura 3), lo que puede estar asociado a la calidad y cantidad de la materia orgánica presente en los suelos volcánicos, la que le confiere una alta capacidad de resiliencia (Dec et al., 2012). Además, los continuos ciclos de humectación y secado, durante el desarrollo del cultivo, provocaron un mayor reordenamiento de las partículas, induciendo a que el suelo sea mas estable a los cambios en su volumen, relacionándose esto a una mayor Da (Dörner et al., 2010b), lo que se refleja en que la $\mathrm{Da}, \mathrm{CA}$ y $\mathrm{k}_{\mathrm{b}}$, en el muestreo SS8 no sean distintos al estado inicial del suelo PD.

\section{CONCLUSIONES}

La preparación de suelo influyó sobre los parámetros medidos, disminuyendo la resistencia mecánica del suelo y la densidad aparente y, como consecuencia, aumentando la capacidad de aire y conductividad de aire. El paso del rodillo compactador causó el efecto contrario: aumentó la resistencia mecánica del suelo y la densidad aparente y, con ello, disminuyó la capacidad de aire y la conductividad de aire.

Los ciclos de humectación y secado influyeron en la estabilización de la porosidad del suelo luego de la labranza, generando valores similares a los que es posible encontrar en el estado original del suelo, tendiendo a aumentar los valores de densidad aparente, mantener los valores de capacidad de aire y aumentar la conductividad de aire.

Los valores de resistencia mecánica variaron principalmente de acuerdo a la dinámica del contenido volumétrico de agua del suelo.

La conductividad de aire superó los valores críticos descritos en la literatura, lo que en este caso podría traducirse en problemas de intercambio gaseoso. Sin embargo, debido a naturaleza dinámica de la estructura del suelo, este recuperó sus atributos funcionales debido a la alta resiliencia descrita para los suelos derivados de cenizas volcánicas.

\section{AGRADECIMIENTOS}

Este trabajo fue financiado por el proyecto FONDECYT 11060130. El primer autor agradece al Dr. José Dörner por la oportunidad, su apoyo y disposición pres- 
tado a este trabajo que se enmarcó dentro del proyecto Fondecyt 11060130 del cual fue responsable. Además, los autores señalados agradecen al Centro Regional de Investigación INIA Remehue por facilitar el espacio físico que permitió el desarrollo del experimento.

\section{REFERENCIAS}

AHMAD, N., UL-HASSAN, F., QADIR, G. 2007. Effect of subsurface soil compaction and improvement measures on soil properties. International Journal of Agriculture \& Biology 9 (3): 509-513.

ALAOUI, A., LIPIEC, J., GERKE, H. 2011. A review of the changes in the soil pore system due to soil deformation: A hydrodynamic perspective. Soil \& Tillage Research 115116: 1-15

ATKINSON, B., SPARKES, D., MOONEY, S. 2009. The impact of soil structure on the establishment of winter wheat (Triticum aestivum). European Journal of Agronomy 30: 243-257.

BACHMANN, J., CONTRERAS, K., HARTGE, K., MACDONALD, R. 2006. Comparison of soil strength data obtained in situ with penetrometer and with vane shear test. Soil \& Tillage Research 87: 112-118.

BALL, B., CAMPBELL, D., DOUGLAS, J., HENSHALL, J. 1997. Soil structural quality, compaction and land management. European Journal of Soil Science 48: 593-601.

BAUMGARTL, T., KÖCK, B. 2004. Modeling volumen change and mechanical properties with hydraulic models. Soil Science Society of America Journal 68: 57-65.

CENTRO DE INFORMACIÓN DE RECURSOS NATURALES (CIREN). 2003. Estudio agrológico X Reg. Tomo 2, CIREN, Santiago.

CZARNES, S., HALLET, P., BENGOUGH A., YOUNG, I. 2000. Root and microbial derived mucilages affect soil structure and water transport. European Journal of Soil Science 51: 435-443.

DEC, D., DÖRNER, J., BALOCCHI, O. 2011. Temporal and spatial variability of structure dependent properties of a volcanic ash soil under pasture in southern chile. Chilean Journal of Agricultural Research 71 (2): 293-303.

DEC, D., DÖRNER, J., BALOCCHI, O., LÓPEZ, I. 2012. Temporal dynamics of hydraulic and mechanical properties of an Andosol under grazing. Soil \& Tillage Research 125: 44-51.

DELTA-T DEVICES LTDA. 2006. User Manual for the SM200 Soil Moisture Sensor. Delta-T Devices Ltda, Cambridge.

DÖRNER, J., HORN, R. 2006. Anisotropy of pore functions in structured Stagnic Luvisols in the Weichselien moraine region in N Germany. Journal of Plant Nutrition and Soil Science 169: 213-220.
DÖRNER, J., SCHROE, V., DEC, D., HORN, R. (eds.). 2009a. Effect of land use on physical properties of a volcanic soil in south Chile. ISTRO 18th Triennial Conference Proceedings, Izmir, Turquía. 15-19 June 2009.

DÖRNER, J., DEC, D., PENG, X., HORN, R. 2009b. Efecto del cambio de uso en la estabilidad de la estructura y la función de los poros de un Andisol (typic hapludand) del sur de Chile. Revista de la Ciencia del Suelo y Nutrición Vegetal 9 (3): 190-209.

DÖRNER, J., DEC, D., PENG, X., HORN, R. 2009c. Change of shrinkage behavior of an Andisol in southern Chile: Effects of land use and wetting/drying cycles. Soil \& Tillage Research 106: 45-53.

DÖRNER, J., DEC, D., PENG, X., HORN, R. 2010a. Effect of land use change on the dynamic behaviour of structural properties of an Andisol in southern Chile under saturated and unsaturated hydraulic conditions. Geoderma 159: 189-197.

DÖRNER, J., SANDOVAL, P., DEC, D. 2010b. The role of soil structure on the pore functionality of an ultisol. Journal of Soil Science and Plant Nutrition 10 (4): 495-508.

DÖRNER, J., DEC, D., ZUÑIGA, F., SANDOVAL, P., HORN, R. 2011. Effect of land use change on Andosol's pore functions and their functional resilience after mechanical and hydraulic stresses. Soil \& Tillage Research 115-116: 71-79.

DÖRNER, J., DEC, D., FEEST, E., VÁSQUEZ, N., DÍAZ, M. 2012. Dynamics of soil structure and pore functions of a volcanic ash soil under tillage. Soil \& Tillage Research 125: 52-60.

ELLIES, A., RAMÍREZ, C., MACDONALD, R. 1993. Variación en la resistencia del suelo por efecto de su uso. Turrialba 43 (1): 77-82.

ELLIES, A., RAMÍREZ, C., MACDONALD, R., FIGUEROA, S. 1994a. Modificaciones estructurales por efecto de tiempo de uso en un Hapludand. Agro Sur 22 (1): 15-22.

ELLIES, A., RAMÍREZ, C., FIGUEROA, S. 1994b. Cambios morfológicos estacionales del espacio poroso de un Hapludand sometido a distintos usos agropecuarios. Agro Sur 22 (1): 23-32.

ELLIES, A., SMITH, R., HORN, R. 1996. Trasmisiones de presiones en el perfil de algunos suelos. Agro Sur 24 (2): 149-158.

ELLIES, A., GREZ, R., RAMÍREZ, C. 1997. La conductividad hidráulica en fase saturada como herramienta para el diagnóstico de la estructura del suelo. Agro Sur 25 (1): 51-56.

ELLIES, A., DÖRNER, J. 1999. Distribución de tensiones en un suelo Palehumult ejercida con el tráfico de la maquinaria agrícola. Agro Sur 27 (2): 1-9.

ELLIES, A., HORN, R., SMITH, R. 2000. Effect of management 
of a volcanic ash soil on structural properties. International Agrophysics 14: 377-384.

FEEST, E. 2011. Efecto de la labranza y ciclos naturales sobre las propiedades hidráulicas de un Andisol durante una temporada de cultivo. Tesis Lic. Agr. Universidad Austral de Chile. 50 p.

FORSYTHE, W. 1974. Manual de laboratorio de física de suelos. Instituto Interamericano de Ciencias Agrícolas de la Organización de los Estados Americanos (OEA). IICA, San José.

HARTGE, K. 2000. The effect of soil deformation on physical soil properties. A discourse on the common background. In: Horn, R., van den Akker, J.J.H., Arvidsson, J. (Eds.), Subsoil compaction-distribution, processes and consequences. Catena Verlag. Reiskirchen, pp. 32-43.

HARTGE, K., HORN, R. 2009. Die physikalische Untersuchung von Bóden. Praxis Messmethoden Auswertung. 4. vollst. Überarbeitete Auflage. Schweizerbart Vorlage, Stuttgart.

HILLEL, D. 1998. Environmental soil physics. 2nd ed. Academic Press. San Diego.

HORN, R. 1988. Compressibility of arable land. In: Drescher, J., Horn, R., de Boodt, M. (Eds.), Impact of water and external forces on soil structure. Catena Supplement 11, pp. 53-71.

HORN, R., BAUMGARTL, T. 1999. Dynamic properties in structured soils. In: Sumner, M. (ed). Handbook of Soil Science. CRC press, Boca Ratón. pp. A19-A52.

HORN, R. 2004. Time dependence of soil mechanical properties and pore functions for arable soils. Soil Science Society of America Journal 68: 1131-1137.

HORN, R., SMUCKER, A. 2005. Structure formation and its consequences for gas and water transport in unsaturated arable and forest soils. Soil \& Tillage Research 82: 5-14.

HORN, R., FLEIGE, H. 2009. Risk assessment of subsoil compaction for arable soils in Northwest Germany at farm scale. Soil \& Tillage Research 102: 201-208.

HUYGENS, D., BOECKX, P., VAN CLEEMPUT, O., OYARZÚN, C., GODOY, R. 2005. Aggregate and soil organic carbon dynamics in South Chilean Andisols. Biogeosciences 2: 159-174.

INSTITUTO DE RECURSOS NATURALES (IREN). 1964. Suelos: Descripciones proyecto aerofotogramétrico Chile/ O.E.A./B.I.D. Publicación N²2. Santiago.

INSTITUTO DE RECURSOS NATURALES (IREN) - Universidad Austral de Chile (UACH). 1978. Estudio de los Suelos de la Provincia de Valdivia. Santiago.

KELLER, T., ARVIDSSON, J., DEXTER, A. 2007. Soil structures produced by tillage as affected by soil water content and the physical quality of soil. Soil \& Tillage Research 92: 45-52. KÉZDI, A. 1980. Handbook of soil mechanics. Soil testing. Vol. 2. Elsevier Publishing Company, Amsterdam.

LEIVA, C. 2009. Caracterización del monto, funcionalidad y evolución del sistema poroso de un Palehumult al ser sometido a ciclos de formación de estructura. Tesis Lic. Agr. Universidad Austral de Chile. 77 p.

MARTÍNEZ, E., FUENTES, J., SILVA, P., VALLE, S., ACEVEDO, E. 2008. Soil physical properties and wheat root growth as affected by no-tillage and conventional tillage systems in a Mediterranean environment of Chile. Soil \& Tillage Research 99: 232-244.

MCVAY, K., BUDDE, J., FABRIZZI, K., MIKHA, M., RICE, C., SCHLEGEL, A., PETERSON, D., SWEENEY, D., THOMPSON, C. 2006. Management effects on soil physical properties in long-term tillage studies in Kansas. Soil Science Society of America Journal 70: 434-438.

MIZOTA, C., VAN REEUWIJK, L. 1989. Clay mineralogy and chemestry of soils formed in volcanic material in diverse climatic regions. International soil reference and information centre. Soil monograph 2. Wageningen.

MOLDRUP, P., YOSHIKAWA, S., OLESEN, T., KOMATSU, T., ROLSTON, D. 2003. Air Permeability in Undisturbed Volcanic Ash Soils: Predictive Model Test and Soil Structure Fingerprint. Soil Science Society of America Journal 67: 32-40.

MORET, D., ARRÚE, J. 2007. Dynamics of soil hydraulic properties during fallow as affected by tillage. Soil \& Tillage Research 96: 103-113.

NAVARRO, A., FIGUEROA, B., ORDAZ, V., GONZÁLEZ, F. 2000. Efecto de la labranza sobre la estructura del suelo, la germinación y el desarrollo del maíz y frijol. Terra 18 (1): 61-69.

NOVOA, R., VILLASECA, S. (eds.). 1989. Mapa agroclimático de Chile. Instituto de Investigaciones Agropecuarias. Santiago.

OSUNBITAN, J., OYEDELE, D., ADEKALU, K. 2005. Tillage effects on bulk density, hydraulic conductivity and strength of a loamy sand soil in Southwestern Nigeria. Soil \& Tillage Research 82: 57-64.

SADZAWKA, A., CARRASCO, M., GREZ, R., MORA, M. 2004. Métodos de análisis recomendados para suelos chilenos. Comisión de Normalización y Acreditación. Sociedad Chilena de la Ciencia del Suelo.

SANDOVAL, M., DÖRNER, J., SEGUEL, O, CUEVAS, J., RIVERA, D. 2012. Métodos de análisis físicos de suelos. Universidad de Concepción. Publicaciones Departamento de Suelos y Recursos Naturales № 5. Chillán.

SCHWEN, A., BODNER, G., SCHOLL, P., BUCHAN, G., LOISKANDL, W. 2011. Temporal dynamics of soil hydraulic 
properties and the water-conducting porosity under different tillage. Soil \& Tillage Research 113: 89-98.

SEGUEL, O., ELLIES, A., MACDONALD, R., RAMÍREZ, C. 2002. Propiedades mecánicas en suelos sometidos a distintos usos. Revista de la Ciencia del Suelo y Nutrición Vegetal 2 (2): 54-61.

SEGUEL, O. 2006. Influencia del potencial mátrico en la generación de estructura y resistencia mecánica de suelos derivados de materiales volcánicos. Tesis Doc. Universidad Austral de Chile. 100 p.

SEMMEL, H., HORN, R., DEXTER A., SCHULZE, E. 1990. The dynamics of soil aggregate formation and the effect on soil physical properties. Soil Technology 3: 113-129.

SIX, J., BOSSUYT, H., DEGRYZE, S., DENEF, K. 2004. A history of research on the link between (micro)aggregates, soil biota, and soil organic matter dynamics. Soil \& Tillage Research 79: 7-31.

SOIL SURVEY STAFF. 2006. Keys to Soil Taxonomy, 10th ed. NRCS, (Agric. Handbk. 436). Washington, DC.

SZŰCS, M., SZŰCS, L., VARGA, Z. 2012. Texture dependence of some physical and water properties of soils in the Danube valley in the Kisalföld region of Hungary I. The bulk density. Acta Agronomica Ováriensis 54 (1): 13-22.

VAN GENUCHTEN, M., LEIJ, F., YATES, S. 1991. The RETC Code for Quantifying the Hydraulic Functions of Unsaturated Soils, Version 1.0. EPA Report 600/2-91/065, U.S. Salinity Laboratory, USDA-ARS, Riverside, California. (On line): <http://www.ars.usda.gov/services/software/ download.htm?softwareid=100> (10 mar. 2013). 
